\title{
BMJ Open Is time to progression associated with post-progression survival in previously treated metastatic non-small cell lung cancer with BRAF V600E mutation? A secondary analysis of phase II clinical trial data
}

Junlong Li, ${ }^{1}$ Medha Sasane, ${ }^{2}$ Jie Zhang, ${ }^{2}$ Jing Zhao, ${ }^{1}$ Marie Louise Ricculli, ${ }^{3}$ Zhiwen Yao, ${ }^{1}$ Suman Redhu, ${ }^{2}$ James Signorovitch ${ }^{1}$

To cite: Li J, Sasane M, Zhang J, et al. Is time to progression associated with post-progression survival in previously treated metastatic non-small cell lung cancer with BRAF V600E mutation? A secondary analysis of phase II clinical trial data. BMJ Open 2018;8:e021642. doi:10.1136/ bmjopen-2018-021642

- Prepublication history and additional material for this paper are available online. To view please visit the journal (http:// dx.doi.org/10.1136/bmjopen2018-021642).

Received 9 January 2018 Revised 29 June 2018 Accepted 17 July 2018

Check for updates

(C) Author(s) (or their employer(s)) 2018. Re-use permitted under CC BY-NC. No commercial re-use. See rights and permissions. Published by BMJ.

${ }^{1}$ Analysis Group Inc., Boston, Massachusetts, USA

${ }^{2}$ Novartis Pharmaceuticals

Corporation, East Hanover, New Jersey, USA

${ }^{3}$ Analysis Group Inc., New York City, New York, USA

Correspondence to

Dr Junlong Li;

junlong.li@analysisgroup.com

\section{ABSTRACT}

Objective Longer time to progression (TTP) is associated with prolonged post-progression survival (PPS) in anaplastic lymphoma kinase+non-small cell lung cancer (NSCLC). This study evaluated whether TTP is associated with PPS among previously treated patients with metastatic v-Raf murine sarcoma viral oncogene homolog B V600E NSCLC receiving dabrafenib as monotherapy or in combination with trametinib.

Design Secondary analysis of phase II clinical trial data. Setting Patients who experienced disease progression treated with dabrafenib monotherapy or in combination with trametinib as second line or later in an open-label, non-randomised, phase II study.

Primary outcome measures The primary outcome was the TTP-PPS association. PPS was assessed with Kaplan-Meier analysis among patients with shorter versus longer TTP ( $<$ or $\geq 6$ months). The TTP-PPS association was quantified in the Cox models adjusting for clinical covariates.

Results of the 84 included patients who progressed on dabrafenib monotherapy $(n=57)$ or combination therapy ( $\mathrm{n}=27), 60$ (71\%) died during post-progression follow-up. Patients with TTP $\geq 6$ months experienced significantly longer PPS compared with those with TTP $<6$ months (median PPS: 9.5 vs 2.7 months, log-rank $p<0.001$ ). Each 3 months of longer TTP was associated with a $32 \%$ lower hazard of death following progression (HR $0.68,95 \%$ $\mathrm{Cl} 0.52$ to 0.88 ) in the multivariable Cox model. Similar associations were seen in each treatment arm.

Conclusion A longer TTP duration after treatment with dabrafenib monotherapy or combination therapy was associated with significantly longer PPS duration. Trial registration number NCT01336634; Post-results.

\section{INTRODUCTION}

Non-small cell lung cancer (NSCLC) accounts for $80 \%-85 \%$ of all lung cancers globally, ${ }^{1}$ and is the leading cause of cancer-related mortality in the USA. ${ }^{2}$ In advanced stages,
Strengths and limitations of this study

- This retrospective study is the first to quantify the association between time to progression (TTP) and post-progression survival (PPS) among previously treated patients with metastatic v-Raf murine sarcoma viral oncogene homolog B V600E non-small cell lung cancer (NSCLC) receiving a new generation of targeted therapies, dabrafenib monotherapy or in combination with trametinib.

- TTP and PPS were chosen as these measures refer to non-overlapping periods of time and yielded prognostic information relevant for physicians considering later-line therapies in advanced NSCLC.

- The TTP-PPS association was estimated using patient-level data from an ongoing pivotal trial via a Cox model, adjusting for multiple patient demographics and disease characteristics.

- The present analysis only included patients who had disease progression observed before death in a clinical trial setting, which may limit the generalisability to other populations.

- The association between TTP and PPS may be confounded by patient characteristics unmeasured in the clinical trial.

NSCLC is aggressive. For example, patients with stage IIIB cancer have an estimated 5 -year survival rate of $5 \%$; this rate is estimated to be about $1 \%$ for patients in stage IV or with confirmed metastatic disease. ${ }^{3}$ Treatment for NSCLC has traditionally consisted of cytotoxic chemotherapy, although recent advances in cancer biology have led to the development of targeted anticancer agents that modulate specific oncogenic molecular pathways. ${ }^{4}$

NSCLC is a heterogeneous cancer, and molecular diagnostic testing can be used 
to inform treatment choice for patients with metastatic or relapsing disease. For example, mutations in v-Raf murine sarcoma viral oncogene homolog B (BRAF), which encodes the protein B-Raf involved in cell growth signalling, are present in $1 \%-5 \%$ of NSCLC. ${ }^{56}$ Constitutively, active B-Raf mutants can prompt tumourigenesis by excessively signalling cells to divide, often via the mitogen-activated protein kinase (MAPK) pathway. ${ }^{7}$ In particular, BRAF V600E mutations account for about $50 \%$ of BRAF mutant NSCLC and $2 \%$ of all NSCLC, and are usually associated with a history of smoking and with adenocarcinoma. ${ }^{8}$ Patients with BRAF V600E mutant NSCLC have poorer clinical outcomes and lower response to platinum-based chemotherapy compared with patients without this mutation. ${ }^{69}$ Thus, targeted therapies that modulate BRAF kinase signalling or downstream MAPK signalling to slow tumour growth are promising alternatives to effectively treat BRAF-mutant NSCLC. ${ }^{10}$

Dabrafenib is a potent and selective reversible BRAF kinase inhibitor, which has previously demonstrated efficacy and tolerability in clinical trials of patients with BRAF V600 mutant melanoma, including those with metastatic disease. ${ }^{11}$ Trametinib, an allosteric inhibitor of mitogen-activated extracellular signal regulated kinase (MEK) 1 and 2, has synergistic antioncogenic activity with BRAF inhibition. The efficacy and tolerability profiles of dabrafenib as a single agent and in combination with trametinib have been assessed among patients with BRAF V600E mutation positive metastatic (stage IV) NSCLC in a recent multicentre, non-randomised, open-label, phase II trial. ${ }^{12}{ }^{13}$ For example, among patients who received at least one prior platinum-based chemotherapy regimen for metastatic disease, patients treated with dabrafenib monotherapy (cohort A) reported an investigator-assessed overall confirmed response rate of $33 \%$ and median progression-free survival (PFS) of 5.5 months. The overall confirmed response rate was $63 \%$ and median PFS was 9.7 months for patients who received dabrafenib and trametinib in combination (cohort B). ${ }^{12} 13$

The overarching goals of NSCLC treatment are to prolong overall survival (OS), manage symptoms and improve patients' quality of life. ${ }^{14}$ However, there are practical challenges to directly assess the effects of treatment on long-term survival in clinical trials of patients with late-stage cancer who have already failed multiple lines of therapy. ${ }^{15}{ }^{16}$ Clinical trials and meta-analyses of other advanced NSCLC treatments have demonstrated that time to progression (TTP) can be predictive of longterm clinical benefits in patient survival. ${ }^{17-19}$ For example, a longer duration of TTP was demonstrated to be significantly associated with a longer duration of post-progression survival (PPS) among patients with NSCLC with anaplastic lymphoma kinase (ALK) gene rearrangement ${ }^{19}$ and mutations in the epidermal growth factor receptor gene. ${ }^{20}$

To the best of our knowledge, the relationship between TTP and PPS has not yet been assessed among patients with BRAF V600E mutant NSCLC receiving the newer generation of targeted therapies. It is of great clinical interest to determine whether any improvement in TTP is offset by loss of survival time in the post-progression period. To address this question, the current study evaluated the association between TTP and the duration of PPS among adult, previously treated, patients with metastatic NSCLC with BRAF V600E mutation who experienced disease progression while receiving dabrafenib monotherapy or in combination with trametinib.

\section{MATERIAL AND METHODS}

\section{Study design and population}

The study is a secondary analysis of data from patients with metastatic NSCLC with BRAF V600E mutation included in the non-randomised, open-label, phase II trial BRF113928. De-identified patient-level data were used in this retrospective analysis. The current analysis included chemotherapy-experienced patients who were assigned to receive either dabrafenib monotherapy $(150 \mathrm{mg}$ two times per day); cohort A) or combination therapy of dabrafenib (150 mg two times per day) and trametinib (2 mg once daily; cohort B) as second-line or later-line and experienced disease progression during the trial's study period. The disease progression was determined based on radiological response as per investigator assessment and Response Evaluation Criteria in Solid Tumours (RECIST) V.1.1. ${ }^{21}$ For patients in cohort B, the study treatment could have been up to the fourth line of systemic anticancer therapy for metastatic disease. The full methodology of this trial has been previously published. ${ }^{12} 13$

A diagram of the patient selection process in the current study is shown in figure 1. Patients, who were previously untreated or did not experience observed disease progression (either due to censoring or death before progression) during the original trial's study period, were excluded from the final analytical sample in this study.

\section{Outcomes and variables}

The primary outcome of interest in the current analysis was PPS which was defined as the time from the date of disease progression after starting the study treatment (dabrafenib monotherapy or combination therapy with dabrafenib and trametinib) until death due to any cause. Patients without an observed death were censored at the date of last contact they were known to be alive. Disease progression was based on radiological response as per investigator assessment and RECIST V.1.1. ${ }^{21}$

The primary independent variable was TTP which was defined as the time from the date of study treatment initiation until the first date of disease progression after treatment initiation. In addition, the following patient characteristics were assessed at baseline: demographics (age, sex and race), history of tobacco use, disease characteristics (Eastern Cooperative Oncology Group (ECOG) performance status before or at the time of progression and time since diagnosis to study treatment initiation), and prior anticancer treatment and response (number of 


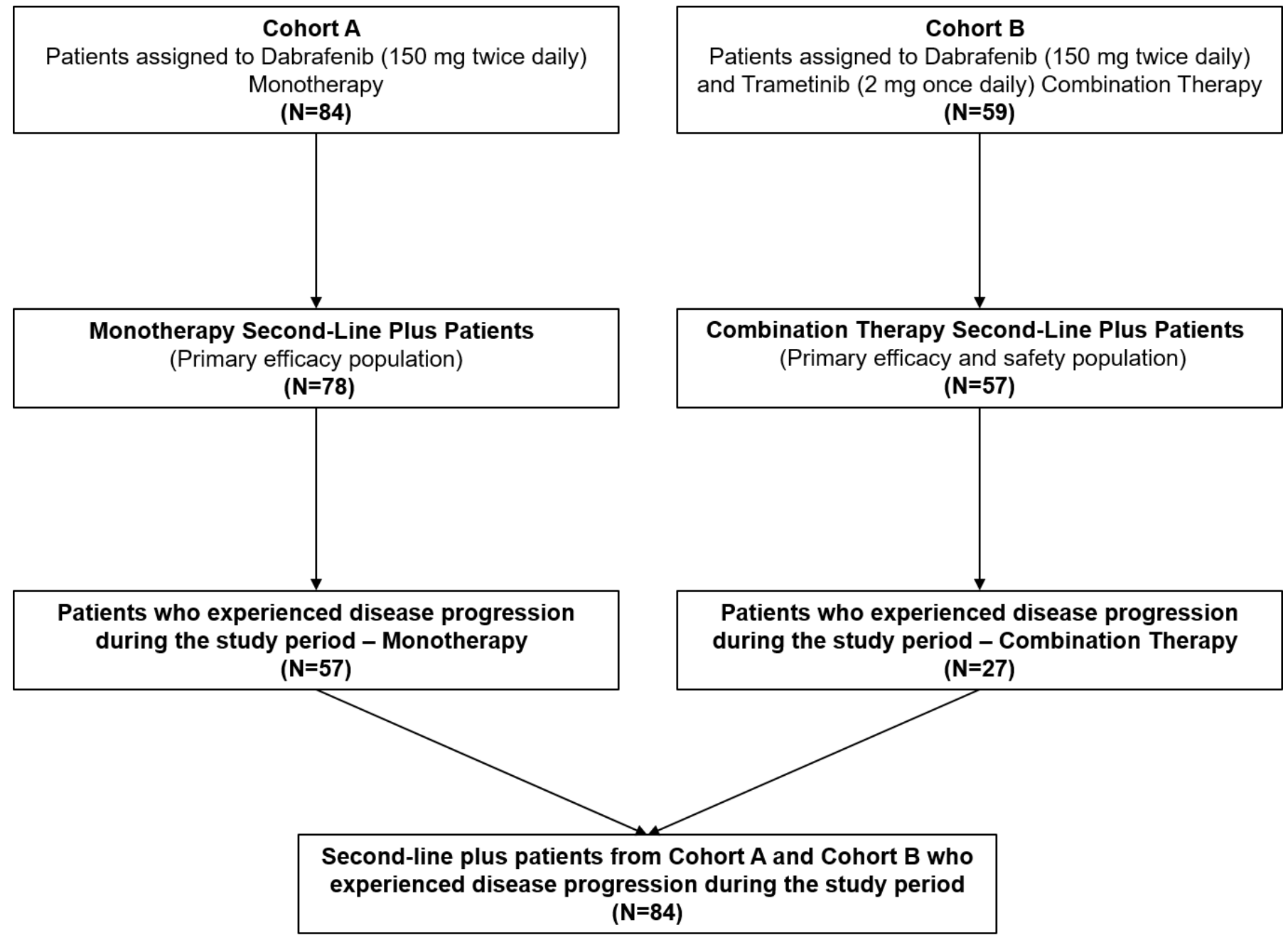

Figure 1 Sample selection flow chart. Patient-level data were used from the non-randomised, open-label, phase II trial BRF113928 (data cut: 7 October 2015, trial ongoing).

prior regimens for metastatic disease, prior radiotherapy, prior maintenance therapy and response to most recent anticancer therapy for metastatic disease).

\section{Statistical analyses}

The association between TTP and PPS was assessed using Kaplan-Meier analysis and a Cox proportional hazards regression analysis. The primary analyses were conducted in the combined cohort of patients who received dabrafenib monotherapy (cohort A) or in combination with trametinib (cohort B). Sensitivity analyses were conducted in each individual cohort and in a subgroup of patients who survived and remained in the trial at 3 months postdisease progression. All analyses were conducted using the statistical software R (V.3.3.2, the $\mathrm{R}$ Foundation for Statistical Computing), and statistical significance was assessed at the $5 \%$ level.

\section{Kaplan-Meier analyses}

To assess the association between TTP and PPS, patients were first categorised into two subgroups based on the length of TTP ( $\geq 6$ months vs $<6$ months) and KaplanMeier curves for PPS were then estimated in each subgroup. The 6-month cut-off in TTP was selected based on the median PFS observed in the combined cohort (ie, 5.3 months), as well as the 'efficacy plateau' observed in median TTP across systemic therapies for advanced NSCLC. ${ }^{9}$

In each subgroup defined by TTP, the number of events (ie, number of patients who died following progression) was summarised, and the median PPS and corresponding 95\% CI were estimated using the Kaplan-Meier method. The log-rank test was used to compare PPS between the two subgroups defined by TTP.

\section{Cox regression analyses}

To further quantify the association between TTP and PPS, a Cox proportional hazards model was implemented. PPS was the time-to-event outcome in the Cox models and TTP was the main independent variable of interest. In this analysis, TTP was a continuous variable as every patient included had progressed on the assigned treatment. Both univariable and multivariable Cox regression analyses were conducted. Prior to analysis, the proportional hazards assumption was tested to ensure the validity of 
the Cox model. The HRs and corresponding 95\% CIs are reported. For clinical relevance, results of the HR associated with TTP are presented for each 3-month increase in TTP.

In the combined cohort, stratified Cox models with cohort as the stratification variable (ie, cohort A (monotherapy) and cohort B (combination therapy)) were conducted as univariable and multivariable regression analyses. The univariable model included TTP as the only independent variable; the multivariable Cox model was further adjusted for the following patient demographics and disease characteristics: age group, sex, race, time since diagnosis to study treatment initiation, history of tobacco use, number of prior regimens for metastatic disease, prior radiotherapy, prior maintenance therapy, response to the most recent prior anticancer therapy and ECOG status before or at progression. ECOG performance was assessed on the date closest to progression to best understand the patients' health status entering into the post-progression period, due to its potential impact on the TTP-PPS association.

In the sensitivity analyses within each cohort, unstratified univariable and multivariable Cox models were used to quantify the TTP-PPS association. The cohort A multivariable Cox model adjusted for the same patient characteristics as those considered in the combined cohort. However, due to a limited sample size and the high proportions of patients in cohort B who were white and had an ECOG score $\leq 1$ before or at progression, these two covariates were not included in the multivariable model for cohort B.

\section{Landmark analysis}

A landmark analysis was conducted as a sensitivity analysis to evaluate the potential for guarantee-time bias in creating the cohorts based on TTP. Patients who died or were censored prior to 3 months after disease progression were excluded. A landmark of 3 months was determined based on a median PPS of 2.7 months among all patients. For patients who were included in the landmark analysis, a Kaplan-Meier analysis and a multivariable Cox regression analysis were conducted using the same approach as described above.

\section{Patient involvement}

No patients were involved in setting the research question or the outcome measures, nor were they involved in the design or conduct of the study. No patients were asked to advise on interpretation or writing up of the results. There are no plans to disseminate the results of the research to study participants.

\section{RESULTS}

\section{Sample selection}

A total of 143 patients with BRAF V600E mutation positive metastatic NSCLC were assigned to receive dabrafenib monotherapy $(n=84$, cohort $A)$ or in combination with trametinib $(\mathrm{n}=59$, cohort $\mathrm{B})$ as second line or above in the BRF113928 trial (figure 1). The final analytical sample comprised 84 patients $(57$ in cohort $\mathrm{A}$ and 27 in cohort B) who actually received the study treatment as second line or above and experienced disease progression during the original trial's study period. The follow-up status of the 51 patients who did not experience disease progression is listed in online supplementary table 1 .

\section{Patient characteristics}

Of the patients in the combined cohort, $50.0 \%$ were male, $48.8 \%$ were under 65 years of age and $79.8 \%$ were white. The majority of patients $(63.1 \%)$ were current or former tobacco smokers. In addition, the mean (SD) time period between diagnosis to the initiation of the study treatment was 21.7 (18.7) months. The proportion of patients with ECOG performance status scores $>1$ before or at progression was $16.7 \%$ overall. It is noteworthy that the ECOG performance status before or at progression was significantly different among patients stratified by TTP (eg, only 1 patient (2.9\%) with TTP $\geq 6$ months had a score $>1$, while 13 patients (26.5\%) with TTP $<6$ months had a score $>1(p=0.01))$. In the combined cohort, the proportions of patients who had received prior radiotherapy or maintenance therapy were $34.5 \%$ and $22.6 \%$, respectively, and $45.2 \%$ of patients had received more than one prior systemic regimen for metastatic disease. The proportion of patients who had achieved either complete or partial response with prior therapy for metastatic disease was $21.4 \%$ (table 1 ). The patient characteristics for each cohort (cohorts A and B) are listed in online supplementary table 2.

\section{Kaplan-Meier analysis of the association between PPS and TTP}

The Kaplan-Meier analysis to assess the association between TTP and PPS among patients in the combined cohort is presented in figure 2. Patients who progressed $\geq 6$ months following treatment initiation experienced significantly prolonged PPS compared with those who progressed before 6 months (log-rank $\mathrm{p}<0.001)$. In the combined cohort, 19 post-progression deaths were observed among 35 patients with TTP $\geq 6$ months, while 41 post-progression deaths were observed among 49 patients with TTP $<6$ months. In addition, these patients with TTP $\geq 6$ months had longer median PPS (9.5 months, 95\% CI 6.6 to 20.2 months) compared with patients with TTP $<6$ months (median PPS: 2.7 months, $95 \%$ CI 1.6 to 4.8 months).

In the sensitivity analysis, a similar association was observed between TTP duration and PPS among cohorts A and B (online supplementary table 3 and figure 1a,b). Specifically, TTP of $\geq 6$ months was associated with fewer deaths and significantly prolonged subsequent survival among patients in each individual cohort (log-rank $\mathrm{p}<0.001$ in cohort A; $\log$-rank $\mathrm{p}=0.026$ in cohort $\mathrm{B})$. 
Table 1 Summary of patient characteristics: combined cohort

\begin{tabular}{|c|c|c|c|c|}
\hline & $\begin{array}{l}\text { Combined } \\
\text { cohort } \\
\mathrm{n}=84\end{array}$ & $\begin{array}{l}\text { Time to } \\
\text { progression } \\
\geq 6 \text { months } \\
\mathrm{n}=35\end{array}$ & $\begin{array}{l}\text { Time to } \\
\text { progression } \\
<6 \text { months } \\
n=49\end{array}$ & P values* \\
\hline \multicolumn{5}{|l|}{ Demographics, n (\%) } \\
\hline Age $<65$ years & $41(48.8)$ & $15(42.9)$ & $26(53.1)$ & 0.48 \\
\hline Male & $42(50.0)$ & $18(51.4)$ & $24(49.0)$ & 1.00 \\
\hline \multicolumn{5}{|l|}{ History of tobacco use, n (\%) } \\
\hline Current or former smoker & $53(63.1)$ & $19(54.3)$ & $34(69.4)$ & 0.24 \\
\hline \multicolumn{5}{|l|}{ Disease characteristics, n (\%) } \\
\hline ECOG performance status before or at progression $>1$ & $14(16.7)$ & $1(2.9)$ & $13(26.5)$ & $0.01 \S$ \\
\hline $\begin{array}{l}\text { No of prior systemic regimens for metastatic disease } \\
>1\end{array}$ & $38(45.2)$ & $14(40.0)$ & $24(49.0)$ & 0.55 \\
\hline Radiotherapy & $29(34.5)$ & $10(28.6)$ & $19(38.8)$ & 0.46 \\
\hline Maintenance therapy & $19(22.6)$ & $8(22.9)$ & $11(22.4)$ & 1.00 \\
\hline \multicolumn{5}{|c|}{ Response to the most recent prior therapy for metastatic disease, $\mathrm{n}(\%) \ddagger$} \\
\hline Complete or partial response & $18(21.4)$ & $5(14.3)$ & $13(26.5)$ & 0.28 \\
\hline
\end{tabular}

*Statistical comparisons were conducted using Wilcoxon rank-sum tests for continuous characteristics and $\chi^{2}$ tests for categorical characteristics.

†Other reported races included Asian, Black or African American and Mixed.

$\S \mathrm{P}<0.05$.

‡Response to the most recent prior anticancer therapy included complete response, partial response, stable disease and progressive disease. Seven patients in cohort A (including one patient with a non-evaluable response) and one patient in cohort B had an unknown response. These patients were imputed as non-complete or partial responders.

ECOG, Eastern Cooperative Oncology Group.

\section{Univariable and multivariable cox regression analyses of the association between PPS and TTP}

In both the univariable and multivariable Cox regression analyses of the association between TTP and PPS, increased duration of TTP was associated with significant reductions in the hazard of post-progression death in the combined cohort. Specifically, each 3-month increase in TTP was associated with a $32 \%$ lower risk of death post-progression in the combined cohort (HR 0.68, $95 \%$ CI 0.57 to $0.83 ; \mathrm{p}<0.001$ ) in the univariable analysis. A similar trend was observed in the multivariable Cox regression analyses conducted to control for patient characteristics that could potentially confound the relationship between TTP and PPS (table 2). In the combined cohort, each 3-month increase in TTP was associated with a $32 \%$ reduction in the risk of post-progression death (HR $0.68,95 \%$ CI 0.52 to $0.88 ; p=0.003)$. In addition to TTP, an ECOG performance score $>1$ before or at progression (HR 3.89, 95\% CI 1.62 to 9.32; $\mathrm{p}=0.002$ ) was also found to be significantly associated with the risk of post-progression death.

Consistent positive TTP and PPS association was demonstrated in each individual cohort in the sensitivity analysis. In the univariable Cox analysis, each 3-month increase in TTP was associated with a $30 \%$ lower risk of post-progression death in cohort A (HR 0.70, 95\% CI 0.57 to $0.88 ; \mathrm{p}=0.001$ ) and a $43 \%$ lower risk in cohort B (HR $0.57,95 \%$ CI 0.34 to $0.97 ; \mathrm{p}=0.035$ ). Each 3-month increase in the duration of TTP was associated with a reduction in the risk of post-progression death by $32 \%$ in cohort A (HR $0.68,95 \%$ CI 0.51 to $0.91 ; p=0.010$ ) and $65 \%$ in cohort B (HR $0.35,95 \%$ CI 0.14 to $0.88 ; \mathrm{p}=0.025$ ) in the multivariable analysis.

\section{Landmark analysis}

Among 84 patients included in the primary analyses, 50 patients who survived and were uncensored from the trial 3 months after their disease progression, were included in the landmark analysis. Using both the Kaplan-Meier approach and the multivariable regression model, results were similar to the primary analysis (online supplementary tables 4 and 5, and supplementary figure 2). The association between TTP and PPS was still positive but not statistically significant due to limited sample size in the landmark analysis (eg, TTP in 3-month increments from the multivariable cox analysis: HR $0.84,95 \%$ CI 0.63 to $1.13 ; \mathrm{p}=0.242$ ). 


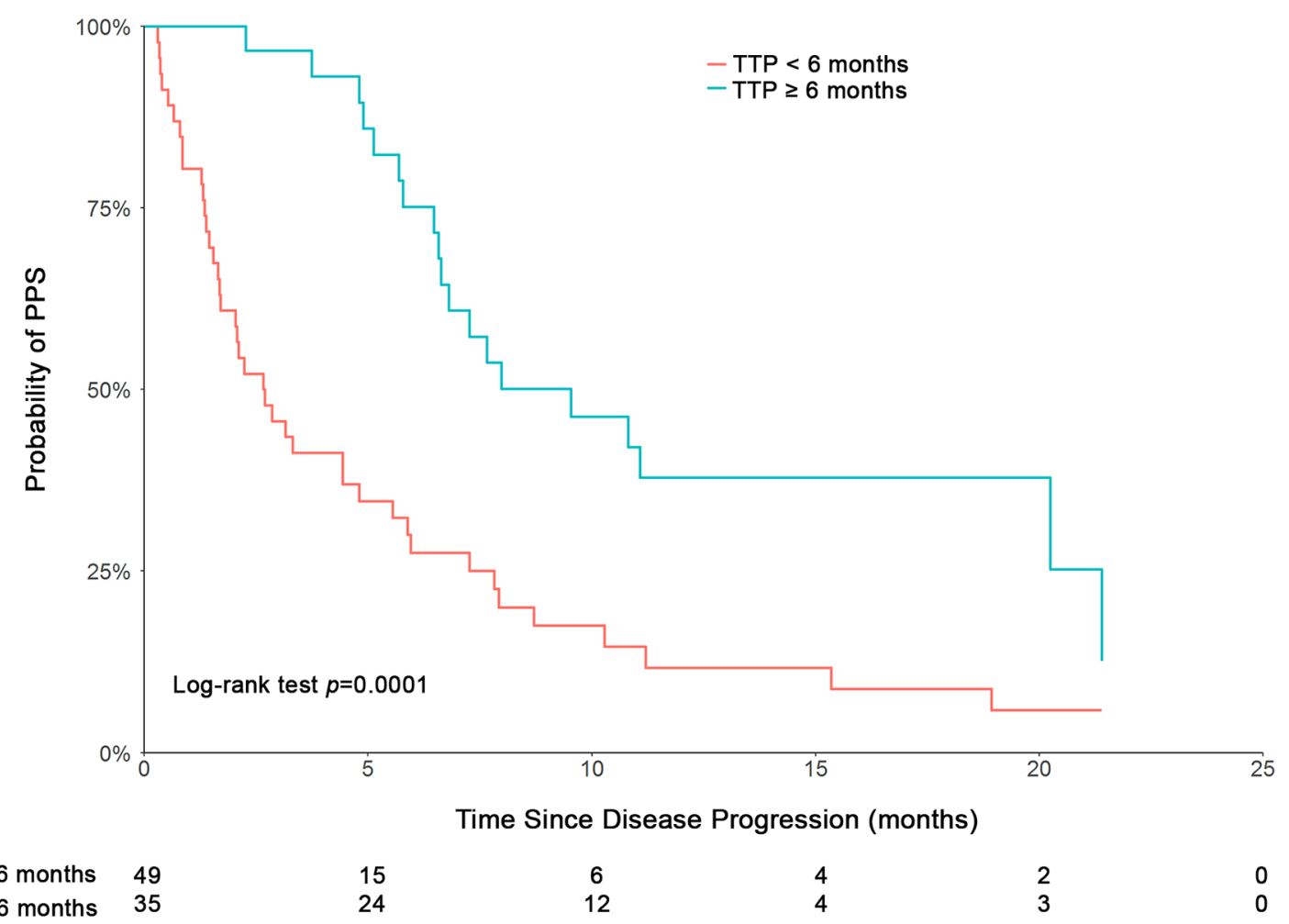

$\begin{array}{llccccc}\text { TTP }<6 \text { months } & 49 & 15 & 6 & 4 & 2 & 0 \\ \text { TTP } \geq 6 \text { months } & 35 & 24 & 12 & 4 & 3 & 0\end{array}$

Figure 2 Kaplan-Meier analysis of PPS in the combined cohort, stratified by duration of TTP. PPS, post-progression survival; TTP, time to progression.

\section{DISCUSSION}

This study addressed the knowledge gap regarding the relationship between TTP and PPS among previously treated patients with metastatic BRAF V600E mutant NSCLC receiving dabrafenib monotherapy or in combination with trametinib who experienced disease progression. The present study quantified the association of TTP-PPS, based on existing patient-level data from the ongoing dabrafenib targeted therapy clinical trial, using a Cox regression model while adjusting for multiple patient demographics and disease characteristics (eg, ECOG score) that may potentially confound the association. ${ }^{12} 13$ The current results indicate the longer a patient objectively benefited from dabrafenib monotherapy or in combination with trametinib (ie, the longer the duration of TTP), the longer the survival period was after objective failure of the targeted therapy (ie, PPS). For every 3-month increase in duration of TTP following treatment

Table 2 The multivariable Cox model of the association between TTP and PPS in the combined cohort

\begin{tabular}{|c|c|c|}
\hline & \multicolumn{2}{|l|}{ Combined cohort } \\
\hline & HR (95\% Cl) & P values \\
\hline Time to progression in 3-month increment(s) & $0.68(0.52$ to 0.88$)$ & $0.003^{*}$ \\
\hline Male vs. female & 0.74 (0.38 to 1.42$)$ & 0.365 \\
\hline Race (white) (yes versus no) & $1.44(0.55$ to 3.76$)$ & 0.462 \\
\hline ECOG performance status before or at progression >1 (yes versus no) & 3.89 (1.62 to 9.32$)$ & $0.002^{*}$ \\
\hline Time since diagnosis to study treatment initiation in 1-month increment(s) & 0.98 (0.96 to 1.00$)$ & 0.058 \\
\hline No of prior systemic regimens for metastatic disease >1 (yes versus no) & $1.22(0.51$ to 2.93$)$ & 0.658 \\
\hline Prior radiotherapy (yes versus no) & $0.88(0.46$ to 1.70$)$ & 0.701 \\
\hline
\end{tabular}

${ }^{*} \mathrm{P}<0.05$

ECOG, Eastern Cooperative Oncology Group; PPS, post-progression survival; TTP, time to progression. 
initiation with dabrafenib monotherapy or in combination with trametinib, patients experienced a $32 \%$ reduction in the hazard of subsequent death after progression when controlling for patient characteristics. In addition, ECOG performance status before or at progression was found to be associated with a significant impact on the association both in the stratified analysis and in the multivariable Cox regression analysis. This result indicates that prolonging TTP with dabrafenib monotherapy or in combination with trametinib is associated with prolongation of OS over and above the longer duration of TTP itself. A consistent positive association between TTP and PPS is observed in the landmark analysis in which patients who died or censored prior to 3 months after disease progression were excluded, although the small sample size may limit the interpretation of these results.

The relationship between TTP and OS in NSCLC has been evaluated and demonstrated to be a moderate to strong association in several studies. ${ }^{22-24}$ However, rather than further explore OS, this study considered the association between TTP and PPS as the primary research question. TTP and PPS were chosen as these measures refer to non-overlapping periods of time and yield prognostic information that can be applied at the time of progression. Also, PPS has been supported as a clinically relevant outcome measure and a valid surrogate endpoint for OS in advanced NSCLC, particularly in evaluations of later-line therapies. ${ }^{25}$ In addition, TTP has been shown to influence PPS in secondary analyses of patients with advanced NSCLC who received first-line chemotherapy or bevacizumab in two clinical trials and an observational cohort study; patients with longer first-line TTP also experienced longer PPS. ${ }^{26}$

With the development of newer therapies for NSCLC, such as targeted therapies that modulate oncogenic molecular pathways active in individuals' tumours, the relationship between TTP and PPS has become a clinically important question for the care of patients with genetic variations. As the first to address this question, Liu et al studied the association between TTP and PPS among patients with advanced NSCLC and ALK mutations who progressed on the targeted therapy of ceritinib. Similar to the results from the current study, a positive association was revealed; every 3 months of longer duration of TTP after initiating ceritinib was associated with a $21 \%$ lower hazard of death following disease progression. It also found that ECOG performance score was another significant predictor for risk of post-progression death, consistent with the finding of the current analysis. ${ }^{19}$ The present study contributes to the evidence that longer duration of TTP is associated with PPS among patients with NSCLC and BRAF V600E mutations receiving targeted therapy who experienced disease progression, and that this association may be a useful way to indicate OS in future clinical trials in this patient population.

This retrospective analysis is subject to several limitations. First, this study should be considered an interim analysis of the association of TTP with PPS in previously treated patients with metastatic BRAF V600E mutant NSCLC. Collection of the progression and survival data in the BRF113928 trial is ongoing. Second, unmeasured patient characteristics could potentially confound the association between TTP and PPS. Other factors such as the use of treatments after progression were not directly included in the present study, and could further affect the TTPPPS association observed in the multivariable Cox regression analyses. In addition, limited sample size within each cohort in the sensitivity analyses may not provide sufficient statistical power to the association assessment. Finally, as the present study only included patients who had disease progression observed before death in a clinical trial setting, these results may not fully generalise to other patient populations.

\section{Conclusions}

In conclusion, a positive relationship between TTP and PPS was demonstrated among adults previously treated for advanced, BRAF V600E mutant NSCLC who received BRAF-specific targeted therapies and experienced disease progression. This relationship was consistent across cohorts with similar patient populations who were treated with dabrafenib monotherapy or in combination with trametinib. This study enriched the understanding and interpretation of TTP-PPS association among patients with metastatic BRAF V600E mutant NSCLC, who were previously treated with at least one platinum-based chemotherapy regimen. Patients who have experienced longer TTP during treatment of dabrafenib monotherapy or in combination with trametinib can expect to experience longer subsequent survival than patients with shorter TTP.

Acknowledgements Medical writing assistance was provided by Shelley Batts and Gloria DeWalt , employees of Analysis Group.

Contributors Conception or design of the work: JL, JieZ, MS and JingZ, JS. Data analysis and interpretation: JL, JieZ, MLR and ZY. Drafting and critical revision of the manuscript: JL, MS, JieZ, JingZ, MLR, SR and JS. Final approval of the version to be published: JL, MS, JieZ, JingZ, MLR, ZY, SR and JS. All authors had, external and internal, had full access to all of the data (including statistical reports and tables) in the study and can take responsibility for the integrity of the data and the accuracy of the data analysis.

Funding This study was funded by Novartis Pharmaceuticals Corporation.

Competing interests JieZ and SR are employees of Novartis Pharmaceuticals Corporation and own stock/stock options. MS was a previous employee of Novartis. JL, JingZ, MLR, ZY and JS are employees of Analysis Group, which has received consultancy fees from Novartis Pharmaceuticals Corporation.

Patient consent Not required.

Ethics approval This study is a secondary analysis of previously published information; no institutional board review was required.

Provenance and peer review Not commissioned; externally peer reviewed.

Data sharing statement The sponsor of the present study, Novartis Pharmaceuticals Corporation, granted permission of the secondary analysis of anonymised clinical trial data. The data were anonymised prior to receipt for the secondary analysis. The datasets generated during and/or analysed during the current study are not publicly available due to clinical trial confidentiality agreement. 
Author note MS formerly at Novartis Pharmaceuticals Corporation.

Open access This is an open access article distributed in accordance with the Creative Commons Attribution Non Commercial (CC BY-NC 4.0) license, which permits others to distribute, remix, adapt, build upon this work non-commercially, and license their derivative works on different terms, provided the original work is properly cited, appropriate credit is given, any changes made indicated, and the use is non-commercial. See: http://creativecommons.org/licenses/by-nc/4.0/.

\section{REFERENCES}

1. Jemal A, Bray F, Center MM, et al. Global cancer statistics. CA Cancer J Clin 2011;61:69-90.

2. American Cancer Society. Cancer Facts and Figures 2017. https:// old.cancer.org/acs/groups/content/@editorial/documents/document/ acspc-048738.pdf.

3. Society AC. Non-small cell lung cancer survival rates. by stage 2016 https://www.cancer.org/cancer/non-small-cell-lung-cancer/ detection-diagnosis-staging/survival-rates.html (accessed April 25, 2017)

4. Capelletto E, Mariniello A, Novello S. Targeted therapy in small cell lung cancer: A new era? Lung Cancer 2017;108:252-3.

5. Brose MS, Volpe P, Feldman M, et al. BRAF and RAS mutations in human lung cancer and melanoma. Cancer Res 2002;62:6997-7000.

6. Cardarella S, Ogino A, Nishino M, et al. Clinical, pathologic, and biologic features associated with BRAF mutations in non-small cell lung cancer. Clin Cancer Res 2013;19:4532-40.

7. Tatematsu T, Sasaki H, Shimizu S, et al. Intra-tumor heterogeneity of BRAF V600E mutation in lung adenocarcinomas. Exp Ther Med 2015:9:1719-22.

8. Paik PK, Arcila ME, Fara M, et al. Clinical characteristics of patients with lung adenocarcinomas harboring BRAF mutations. J Clin Oncol 2011;29:2046-51.

9. Marchetti A, Felicioni L, Malatesta S, et al. Clinical features and outcome of patients with non-small-cell lung cancer harboring BRAF mutations. J Clin Oncol 2011;29:3574-9.

10. Huang T, Karsy M, Zhuge J, et al. B-Raf and the inhibitors: from bench to bedside. J Hematol Oncol 2013;6:30.

11. Gibney GT, Zager JS. Clinical development of dabrafenib in BRAF mutant melanoma and other malignancies. Expert Opin Drug Metab Toxicol 2013;9:893-9.

12. Planchard D, Besse B, Groen HJM, et al. Dabrafenib plus trametinib in patients with previously treated BRAF(V600E)-mutant metastatic non-small cell lung cancer: an open-label, multicentre phase 2 trial. Lancet Oncol 2016;17:984-93.

13. Planchard D, Kim TM, Mazieres J, et al. Dabrafenib in patients with BRAF(V600E)-positive advanced non-small-cell lung cancer: a single-arm, multicentre, open-label, phase 2 trial. Lancet Oncol 2016;17:642-50.

14. American Society of Clinical Oncology. Systemic Therapy for Stage IV Non-Small Cell Lung Cancer Cancer.net. 2015 http://www. cancer.net/research-and-advocacy/asco-care-and-treatmentrecommendations-patients/systemic-therapy-stage-iv-non-smallcell-lung-cancer (accessed 25 Apr 2017).

15. Korhonen $\mathrm{P}$, Zuber $\mathrm{E}$, Branson $\mathrm{M}$, et al. Correcting overall survival for the impact of crossover via a rank-preserving structural failure time (RPSFT) model in the RECORD-1 trial of everolimus in metastatic renal-cell carcinoma. J Biopharm Stat 2012;22:1258-71.

16. Miller VA, Hirsh V, Cadranel J, et al. Afatinib versus placebo for patients with advanced, metastatic non-small-cell lung cancer after failure of erlotinib, gefitinib, or both, and one or two lines of chemotherapy (LUX-Lung 1): a phase $2 \mathrm{~b} / 3$ randomised trial. Lancet Oncol 2012;13:528-38.

17. Pilz LR, Manegold C, Schmid-Bindert G. Statistical considerations and endpoints for clinical lung cancer studies: Can progression free survival (PFS) substitute overall survival (OS) as a valid endpoint in clinical trials for advanced non-small-cell lung cancer? Trans/ Lung Cancer Res 2012;1:26-35.

18. Fleischer F, Gaschler-Markefski B, Bluhmki E. A statistical model for the dependence between progression-free survival and overall survival. Stat Med 2009;28:2669-86.

19. Liu G, Zhang J, Zhou ZY, et al. Association between time to progression and subsequent survival inceritinib-treated patients with advanced ALK-positive non-small-cell lung cancer. Curr Med Res Opin 2016;32:1911-8.

20. Yoshino R, Imai H, Mori K, et al. Surrogate endpoints for overal survival in advanced non-small-cell lung cancer patients with mutations of the epidermal growth factor receptor gene. Mol Clin Oncol 2014;2:731-6.

21. Eisenhauer EA, Therasse $P$, Bogaerts J, et al. New response evaluation criteria in solid tumours: revised RECIST guideline (version 1.1). Eur J Cancer 2009;45:228-47.

22. Hotta K, Fujiwara Y, Matsuo K, et al. Time to progression as a surrogate marker for overall survival in patients with advanced nonsmall cell lung cancer. J Thorac Oncol 2009;4:311-7.

23. Imai $\mathrm{H}$, Takahashi $\mathrm{T}$, Mori $\mathrm{K}$, et al. Individual-level data on the relationships of progression-free survival, post-progression survival, and tumor response with overall survival in patients with advanced non-squamous non-small cell lung cancer. Neoplasma 2014;61:233-40.

24. Imai H, Mori K, Wakuda K, et al. Progression-free survival, postprogression survival, and tumor response as surrogate markers for overall survival in patients with extensive small cell lung cancer. Ann Thorac Med 2015;10:61-6.

25. Kotake M, Miura $\mathrm{Y}$, Imai $\mathrm{H}$, et al. Post-Progression Survival Associated with Overall Survival in Patients with Advanced NonSmall-Cell Lung Cancer Receiving Docetaxel Monotherapy as Second-Line Chemotherapy. Chemotherapy 2017;62:205-13.

26. Langer $\mathrm{C}$, Wozniak A, Socinski M, et al. Does time to progression (TTP) influence post-progression survival (ppOS)?: Analyses from the PointBreak, ECOG 4599, and ARIES studies of bevacizumab in non-small cell lung cancer (NSCLC). J Clin Oncol 2015;33. abstr e19019 